Psychother Psychosom 2019;88:244-246

DOI: $10.1159 / 000500108$

\section{Long-Term Effectiveness of Cognitive Therapy for Refractory Social Anxiety Disorder: One-Year Follow-Up of a Randomized Controlled Trial}

\author{
Naoki Yoshinaga ${ }^{\mathrm{a}}$ Kazumi Kubota $^{\mathrm{b}}$ Kensuke Yoshimura ${ }^{\mathrm{c}}$ \\ Rieko Takanashid ${ }^{\text {Yasushi Ishida }}{ }^{\mathrm{e}}$ Masaomi lyo $^{f}$ \\ Takashi Fukudag Eiji Shimizu ${ }^{\mathrm{d}, \mathrm{h}}$ \\ a Organization for Promotion of Tenure Track, University \\ of Miyazaki, Miyazaki, Japan; b Department of Biostatistics, \\ Yokohama City University School of Medicine, Yokohama, Japan; \\ 'Health Care Management Center, Chiba University Hospital, \\ Chiba, Japan; 'Department of Cognitive Behavioral Physiology, \\ Graduate School of Medicine, Chiba University, Chiba, Japan; \\ e Division of Psychiatry, Department of Clinical Neuroscience, \\ Faculty of Medicine, University of Miyazaki, Miyazaki, Japan; \\ ${ }^{f}$ Department of Psychiatry, Graduate School of Medicine, \\ Chiba University, Chiba, Japan; ${ }^{9}$ Center for Outcomes Research \\ and Economic Evaluation for Health, National Institute of \\ Public Health, Wako, Japan; ${ }^{h}$ Research Center for Child Mental \\ Development, Chiba University, Chiba, Japan
}

\section{Dear Editor,}

There is a large body of evidence that antidepressants and cognitive therapy (CT) are beneficial for the treatment of social anxiety disorder (SAD) [1]. Although individual CT has recently been regarded as the first-choice treatment for $\mathrm{SAD}$, antidepressants are still commonly used in clinical practice $[2,3]$. Therefore, more work is required to garner evidence of next-step treatment options for SADs who have been ineffectively treated with antidepressants.

Only four studies have assessed augmentation or switching strategies for SADs who were refractory to antidepressants [4-7]. Among these next-step strategies, individual CT as an adjunct to usual care has achieved the highest rates of treatment response $(85.7 \%)$ and remission (47.6\%) at the end of treatment [4]. Conversely, other medication augmentation/switching strategies had response rates of $48.3-70.0 \%$ and remission rates of $18.6-27.0 \%$ [5-7]. However, these studies have only assessed short-term treatment outcomes (the longest being 24 weeks).

In this paper, we report on 1-year follow-up data evaluating the long-term effectiveness of individual CT in addition to usual care for antidepressant-refractory SAD in our previous randomized controlled trial [4], focusing on the adjunctive CT group. We supplementarily evaluated the cost-effectiveness of adjunctive CT, but the details are presented only in the online supplementary material (online suppl. Appendix; for all online suppl. material, see www.karger.com/doi/10.1159/000500108).

\section{KARGER}

E-Mail karger@karger.com www.karger.com/pps
(C) 2019 The Author(s)

Published by S. Karger AG, Basel

Karger
Open access

This article is licensed under the Creative Commons AttributionNC-ND) (http://www.karger.com/Services/OpenAccessLicense) Usage and distribution for commercial purposes as well as any distribution of modified material requires written permission. NonCommercial-NoDerivatives 4.0 International License (CC BY-
Eligible antidepressant-refractory SADs $(n=42)$ were randomly allocated to two groups to either receive individual CT in addition to usual care (adjunctive CT, $n=21$ ) or usual care alone (UC alone, $n=21$ ). Local primary psychiatrists referred patients to the trial but continued to provide usual care to patients in both groups. Seven therapists ( 4 clinical psychologists, 1 psychiatrist, 1 registered nurse, and 1 psychiatric social worker) delivered 16 individual weekly CT sessions based on the Clark and Wells model. Both groups received active interventions for 16 weeks, but thereafter, participants in the UC alone group were offered individual CT if desired (62\% subsequently received individual CT; that data is not reported here).

The primary outcome was the severity level of social anxiety, using the Liebowitz Social Anxiety Scale-Self Report (LSAS-SR). Secondary self-reported outcome measures: Patient Health Questionnaire-9 item (PHQ-9), Generalized Anxiety Disorder-7 item (GAD-7), and EuroQol-5 Dimensions (EQ-5D). Data was collected for both groups at week 0 (pre-intervention: Pre), week 8 (midintervention: Mid), and week 16 (post-intervention: Post). For the adjunctive CT group, data was collected at week 20 (1-month follow-up: 1M-FU), week 28 (3-month follow-up: 3M-FU), week 40 (6-month follow-up: 6M-FU), and week 64 (1-year follow-up: 1YFU).

All analyses were performed using JMP 12 PRO (SAS Institute), and a two-tailed alpha level of 0.05 was used to define statistical significance. To assess long-term effectiveness of adjunctive CT, mean total scores at each assessment point for all outcomes were analyzed with single-factor (time) repeated measures analysis of variance (ANOVA). Where repeated measures ANOVA indicated significant changes, pairwise $t$ tests were calculated post hoc. Primary analyses were based on the intent-to-treat principle without imputing missing data. For sensitivity analysis, the linear mixed model was also applied to examine effects of missing data.

There were no significant group differences in baseline clinical characteristics (see original trial [4]). Concerning attrition, one patient in the UC alone group dropped out during the short-term intervention period due to a worsening of depressive symptoms, and three patients in the adjunctive CT group dropped out during the follow-up period ( 1 moved, 1 unwilling to continue, and 1 work-related schedule conflict).

As shown in Figure 1, all primary and secondary outcomes in the adjunctive CT group were significantly improved by week 16 (Post), and observed improvements were maintained until the end of the follow-up (1M-FU). Two of the four measures (LSAS-SR and PHQ-9) showed significant improvements from Pre to Post and from Post to 1Y-FU. On the primary outcome (LSAS-SR), the Pre to Post and Pre to 1Y-FU within-group effect sizes of adjunctive CT were $\mathrm{d}=1.91$ and $d=2.66$, respectively. At the $1 \mathrm{Y}$-FU assessment, $85.7 \%$ of patients in the adjunctive CT group were judged to be responsive to the treatment $(18 / 21$, three dropped out), and $57.1 \%$ met the remission criteria $(12 / 21)$. When carrying

Naoki Yoshinaga

Organization for Promotion of Tenure Track

University of Miyazaki

5200 Kiyotake, Kihara, Miyazaki-city, Miyazaki 889-1692 (Japan)

E-Mail naoki-y@med.miyazaki-u.ac.jp 


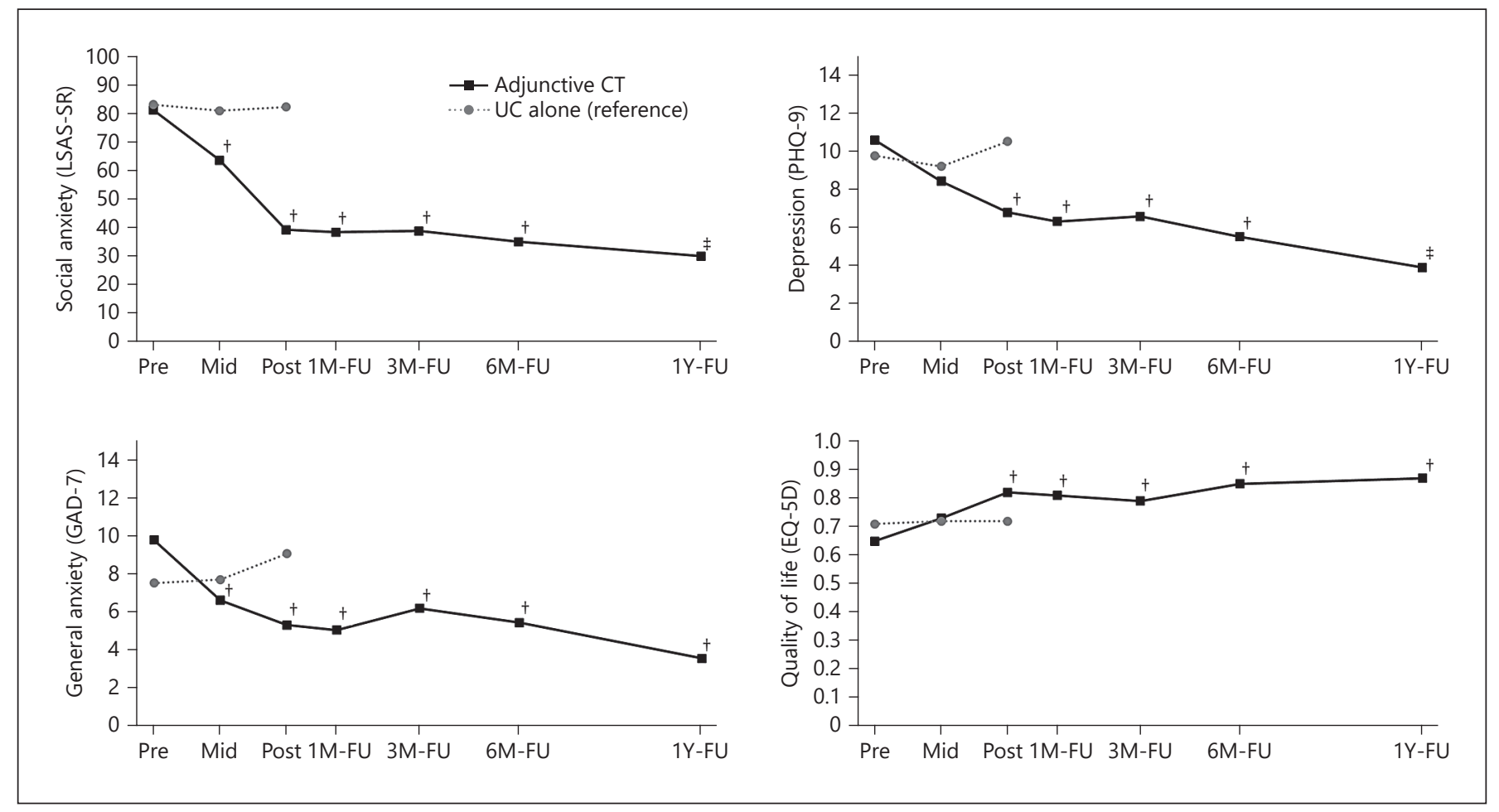

Fig. 1. Observed long-term outcome data in the adjunctive CT group. ${ }^{\dagger} p<0.05$, significant difference in pairwise comparisons with week $0 .{ }^{\ddagger} p<0.05$, significant difference in pairwise comparisons with both weeks 0 and 16 . Higher scores on the EQ-5D indicate better quality of life. Higher scores on the other measures indicate greater pathology or severity.

the last observation forward for dropouts, these percentages were 95.2\% (responded) and 57.1\% (remitted). See online supplementary Results for more details.

The findings of this study indicate that the effects of adjunctive CT seemed to be well maintained 1 year after completion of treatment for measures of social anxiety, depression, generalized anxiety, and quality of life. The Pre to 1Y-FU treatment effect size of adjunctive CT was large $(d=2.66)$. The course of improvement demonstrates that antidepressant-refractory SADs exhibit substantial treatment gains immediately following treatment and that these are well maintained at the 1-month, 3-month, 6-month, and 1 -year follow-up points. These results are consistent with the favorable long-term effects of individual CT for SADs observed in previous studies also carried out over at least 1 year, which did not target antidepressant-refractory patients [e.g., 8-10]. Thus, the findings of our study underscore the long-term benefits of adjunctive CT in improving social anxiety and other related symptoms, even in antidepressant-refractory cases.

The main limitations of the study are: (a) small sample size, (b) lack of follow-up clinical outcome data in the UC alone group, and (c) lack of follow-up information regarding concurrent medication in both groups. We also could not determine whether adjunctive CT itself, or other non-treatment-specific factors, contributed to greater clinical effectiveness. The current study intended to examine the additional value of CT to usual care but was not an examination of the effectiveness of CT itself. To evaluate outcomes with more confidence, a replication study is needed which employs a larger sample and obtains complete follow-up data including concurrent medication information. It would also be of interest to directly compare individual CT with other intervention-delivery methods (e.g., group based, internet based), with other forms of psychotherapy, and/or with other medications.

In conclusion, the results of this study suggest that adding CT to usual care for antidepressant-refractory SAD can produce substantial, maintained effects.

\section{Acknowledgement}

The authors would like to express their gratitude to Dr. Mari Tanaka, Ms. Hanae Ibuki, Dr. Keiko Ohshiro, Dr. Fumiyo Ohshima, Dr. Kenichi Asano, and Dr. Satoshi Matsuki for providing the treatment, collecting data, and/or screening patients, to Dr. Hideki Hanaoka, Dr. Yasunori Sato, and Dr. Masaya Koshizaka for managing and monitoring the trial, and to Mr. Richard White for checking the English language.

\section{Statement of Ethics}

The study protocol has been reviewed and approved by the ethics committee at the Chiba University Graduate School of Medicine (reference number: 1422) and carried out in accordance with the World Medical Association Declaration of Helsinki. Written informed consent was obtained from all patients after the procedures had been fully explained. 


\section{Disclosure Statement}

N.Y. has received writing honoraria from Igaku-Shoin, Medical Friends, Nihon-Hyouronsha, and Sogensha. R.T. has received a speaking honorarium from Mitsubishi Tanabe Pharma and writing honorarium from Chugaiigakusha. The other authors declare no competing interests.

\section{Funding Sources}

This study was supported by the Grant-in-Aid for the Japan Society for the Promotion of Science (JSPS) Fellows from the JSPS (grant 13J00177 to N.Y.), part of the Grant for Scientific Research on Priority Areas from the University of Miyazaki (to N.Y.), part of the Grant-in-Aid for Scientific Research from the Japanese Ministry of Health, Labor and Welfare (grant 22SE1P0051 to Yutaka Ono and E.S.), and part of the Special Budget for Projects from the Japanese Ministry of Education, Culture, Sports, Science and Technology (to E.S.). The funders had no role in the acquisition or analysis of the data or the content of this article.

\section{Author Contributions}

N.Y., K.Y., M.I., T.F., and E.S. conceptualized and designed this study. M.I. and E.S. coordinated the trial. N.Y. and R.T. collected data. E.S. administered supervised therapies and performed patients' screening. N.Y., K.K., K.Y., and T.F. substantially contributed to the data analysis, and all authors to the interpretation of the results. N.Y. and K.K. drafted the first manuscript, and all authors critically revised the first version for important intellectual content. All authors read and approved the final manuscript.

\section{References}

1 Stein MB, Stein DJ. Social anxiety disorder. Lancet. 2008 Mar;371(9618): 1115-25.

2 Wong J, Motulsky A, Eguale T, Buckeridge DL, Abrahamowicz M, Tamblyn R. Treatment indications for antidepressants prescribed in primary care in Quebec, Canada, 2006-2015. JAMA. 2016 May;315(20):2230-2.

3 Huijbregts KM, Hoogendoorn A, Slottje P, van Balkom AJ, Batelaan NM. Long-term and short-term antidepressant use in general practice: data from a large cohort in the Netherlands. Psychother Psychosom. 2017; 86(6):362-9.

4 Yoshinaga N, Matsuki S, Niitsu T, Sato Y, Tanaka M, Ibuki H, et al. Cognitive behavioral therapy for patients with social anxiety disorder who remain symptomatic following antidepressant treatment: a randomized, assessor-blinded, controlled trial. Psychother Psychosom. 2016;85(4): 208-17.

5 Van Ameringen M, Mancini C, Wilson C. Buspirone augmentation of selective serotonin reuptake inhibitors (SSRIs) in social phobia. J Affect Disord. 1996 Jul;39(2):115-21.

6 Pallanti S, Quercioli L. Resistant social anxiety disorder response to Escitalopram. Clin Pract Epidemiol Ment Health. 2006 Dec;2(1):35.

7 Pollack MH, Van Ameringen M, Simon NM, Worthington JW, Hoge EA, Keshaviah A, et al. A double-blind randomized controlled trial of augmentation and switch strategies for refractory social anxiety disorder. Am J Psychiatry. 2014 Jan;171(1):44-53.

8 Stangier U, Schramm E, Heidenreich T, Berger M, Clark DM. Cognitive therapy vs interpersonal psychotherapy in social anxiety disorder: a randomized controlled trial. Arch Gen Psychiatry. 2011 Jul;68(7):692-700.

9 Leichsenring F, Salzer S, Beutel ME, Herpertz S, Hiller W, Hoyer J, et al. Long-term outcome of psychodynamic therapy and cognitive-behavioral therapy in social anxiety disorder. Am J Psychiatry. 2014 Oct;171(10): 1074-82.

10 Nordahl HM, Vogel PA, Morken G, Stiles TC, Sandvik P, Wells A. Paroxetine, cognitive therapy or their combination in the treatment of social anxiety disorder with and without avoidant personality disorder: a randomized clinical trial. Psychother Psychosom. 2016;85(6):346-56. 